\title{
A critical reflection on the discipline of nursing based on the correct analysis of the relationship between medicine and nursing ${ }^{\dagger}$
}

Review

Rui-Fang Zhuª, Hong-Xia Rena, Xiu-Juan Wanga, Chi-Chen Zhang ${ }^{b}$, Qi Yub, Zhi-Guang Duan,*, Shi-Fan Han ${ }^{a, *}$

${ }^{a}$ School of Nursing, Shanxi Medical University, Taiyuan, Shanxi 030001, China

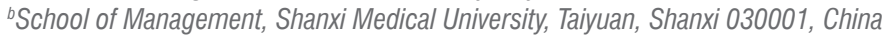

Received: 13 September 2018; Accepted: 30 November 2018; Published: 20 March 2019

Abstract: As nursing becomes a first-level discipline, the central focus of the development process of nursing science is the independent exploration of the future path of nursing care. Although many countries have made efforts to develop nursing as an independent discipline, no remarkable advancement has been achieved due to the incorrect understanding of the relationship between nursing and medicine and the improper comprehension of other factors involved in the process of nursing reform. Teaching and practicing nursing also face many problems. Based on our understanding of the relationship between nursing and medicine and through a careful analysis of the existing problems in the current nursing discipline, this paper provides some insights into the future directions of nursing development by exploring the independently developing nursing discipline in foreign countries.

Keywords: medicine $\bullet$ nursing $\bullet$ reflection $\bullet$ nursing discipline $\bullet$ nursing theory

(c) Shanxi Medical Periodical Press

\section{The relationship between medicine and nursing}

Professor of Psychiatry and Internal Medicine at the University of Rochester, George L. Engel, once proposed that "in order to understand the determining factors of a disease and to establish rational treatment and health care models, the medical model must also consider the social environment of the patient, the patient's life, and

'This project was supported by the hospital foundation of the First Hospital of Shanxi Medical University of China (no. YH161705). the complementary system that the society decides to use to deal with the devastating effects of the disease, that is, the role of doctors and the health care systems". ${ }^{1}$ In the future, nursing care will extend to the entire person, the entire process, the entire family, and the entire society. As researchers in nursing science in the new era, we often reflect on the relationship between medicine and nursing. What are the current problems in the nursing discipline, and what is the trend of the development of the nursing discipline?

Nursing is an ancient art. Since the dawn of human beings, nursing activities have existed. Previously,

How to cite this article: Zhu RF, Ren HX, Wang XJ, et al. A critical reflection on the discipline of nursing based on the correct analysis of the relationship between medicine and nursing. Front Nurs. 2019; 1: xx-xx. 
nursing was a second-level discipline associated with clinical medicine. In 2011, in consideration of the needs of "Nursing science" development internationally and the specific status of undergraduate nursing education in China at that time, the Academic Degrees Committee of the Chinese State Council finally repositioned nursing science into a first-level science/discipline after repeated debates by experts from the Academic Degree and Graduate Education Committee of China.

Although nursing has become a first-level discipline, medical professionals are still constantly debating about the relationship between nursing and medicine, particularly, the relationship between clinical medicine and nursing care. From a philosophical point of view, the relationship of mutual dependency can be divided into sequential dependency, interdependency, NorthSouth dependency, and deterministic dependency. Interdependency refers to the inseparable interaction between one subject and another, indicating that when one of these subjects exists, the other must also exist. If one subject disappears, then the other also loses its meaning of existence. From that perspective, medicine and nursing have an interdependent relationship and are mutually dependent on one another. Ancient nursing care in China focused on " $30 \%$ treatment and $70 \%$ nursing", in which "nursing" indicates patient care; similarly, today's clinical medicine is composed of $30 \%$ treatment and $70 \%$ nursing care, suggesting that nursing has played a very important role in medicine from early on. In the nursing discipline, $70 \%$ of the workload comprises physical and psychological care, and 30\% comprises medical treatment. Therefore, medicine and nursing are interdependent and mutually indispensable.

Although medicine and nursing have an interdependent relationship, they are also distinctive from one another. Clinical medicine focuses specifically on the diagnosis and treatment of diseases; thus, the learned clinical aspects are rigorous. Conversely, nursing requires direct contact with patients every day, and all aspects beyond medical treatment that are essential for a patient's recovery and peaceful discharge from the hospital are performed by nurses.

\section{Existing problems in the nursing discipline}

Education is the foundation of a country. Good nursing education is not only a necessary prerequisite for clinical nursing practice and teaching in nursing, but it is also required for nursing management and research work in nursing science. As an independent discipline, nursing demands independent thinking and a separate educational system, theoretical system, and so on. The following issues that currently exist in nursing will be discussed from the aspects of theoretical education and practical education.

\subsection{Theoretical knowledge}

\subsubsection{Curriculum}

The current nursing education curriculum in China can be divided into three categories: (1) general public courses, such as physical education, college English, and so on; (2) basic medical courses, such as physiology, biochemistry, anatomy, pharmacology, and so on; and (3) nursing professional courses, such as General Nursing, Internal Medicine, Surgery, Obstetrics and Gynecology, Pediatrics, Community Nursing, and so on. The following areas have existing problems.

(1) Course contents: Nursing education, in terms of both theory and knowledge, remains under the influence of previous biomedical models. The course adopts a three-stage approach. Most undergraduate colleges have gradually replaced "Diagnostics", the clinical textbook in medical science once used by nursing majors, with "Health Assessment", 2,3 which can weaken the influence of nursing students' medical thinking and impede the establishment of a complete medical system that requires nurses to understand the basic concept of medicine. "Diagnostics" is one of the most important fundamental courses for medical students, which bridges the medical education system between nursing and medicine. It is particularly important for developing clinical thinking and practical abilities in the students. "Diagnostics" focuses on the understanding and recognition of diseases. It can provide guidance for disease diagnosis and confirmation of treatment measures, which is conducive to the clinical triage division of nursing students. In addition to the basic skills that are required during clinical practice, modern nurses should also possess a certain degree of general knowledge in psychological care and the concept of humanistic care. These humanities courses are not listed as mandatory courses but are elective courses in Chinese institutes of higher education. The current curriculum may produce students who lack humanistic concepts and neglect the humanity aspect in nursing care. ${ }^{4}$ Some institutions have assigned the humanities curriculum as a mandatory course. However, instructors who teach the course have no nursing background and only specialize in humanities studies. These teachers 
can only teach the theory to the students without any real experience in clinical practice. Thus, taking the course does not help the students obtain first-hand experience or understanding; they only learn confusing theories, and the concepts cannot be fully comprehended or applied in the future nursing careers of the student. Regardless, general humanities courses, nature-related courses, and social science courses are widely offered at universities in Denmark, Australia, the United States, and other countries. . $^{5}$

(2) Curriculum arrangement: Most of the undergraduate nursing curriculums in Chinese educational institutes begin with general medicine. Basic physiology and biochemistry courses are taught before beginning the nursing professional courses. Conversely, in other countries, courses such as "Introduction to Nursing" are offered from the beginning of enrollment so that students can have a general understanding of nursing to decide whether to continue to pursue a nursing major. Moreover, in the nursing education plan of those countries, humanities courses are taught at the beginning of the new school year or at the start of the second semester, with the intention that the students will remember the concept of humanistic care that they learned from the beginning of the school year. ${ }^{5,6}$ However, the undergraduate nursing education curriculum in China lacks these systematic arrangements. For example, redundant knowledge is repeated in the internal medicine and surgery courses. The current textbooks and teaching require better integration with one another to avoid overlapping information. ${ }^{7}$ In addition, nursing science education in China has greatly emphasized medical science, whereas the international nursing education program curriculum is not biased toward medicine. Instead, it begins with health and then extends to involve all other areas of health, including physiological, psychological, spiritual, cultural, social, behavioral, and the environmental factors that affect an individual's health.

\subsubsection{Training objectives}

Currently, based on the nursing training objectives, the College of Nursing of Jilin University has set goals in four areas of student education: concepts, knowledge, abilities, and quality. The School of Nursing of Central South University has proposed training requirements for quality, knowledge, and skills. The Nanjing Medical University School of Nursing proposed seven specific training objectives. Li et al. ${ }^{7}$ proposed three educational goals after studying the training objectives of undergraduate nursing courses in China: knowledge, ability, and quality.

However, many problems exist in these training objectives. The National Bureau of Education noted that for professional nursing certification, a nursing program should cultivate critical thinking abilities, solid theoretical knowledge, strong practical abilities, and scientific research abilities in their nursing students. ${ }^{8}$ However, although our undergraduate nursing education programs have greatly emphasized the learning of theoretical knowledge in the curriculum setting, they have not paid sufficient attention to courses of practical value such as statistics. Moreover, undergraduate medical students can graduate without a thesis defense requirement, which further weakens the ability of most students to conduct scientific research. Furthermore, undergraduate nursing students are not required to take a diagnostics course, which is a major reason why nurses do not have the right to prescribe medication. Under this circumstance, we may want to reflect on whether nursing education under the guidance of a medical model is a better choice for the discipline.

\subsubsection{The faculty team}

Nursing science education aims to train students to gain a solid theoretical foundation, practical ability, scientific research ability, humanistic qualities, as well as strong evaluation and observation abilities. The faculty team in nursing education plays critical roles in achieving these goals. Presently, the personnel engaged in nursing professional teaching in Chinese colleges and universities can be roughly divided into three groups: (1) the specialized nursing teachers who are no longer clinical practitioners; (2) clinicians who also serve as nursing faculties; and (3) senior head nurses who have extensive clinical experience. The advantage of having clinicians as teaching faculties for the nursing major lies in their strong background in medical theories. However, because the doctors are not graduates from the nursing major, their teaching may lack insight into nursing care. Specialized nursing teachers often have solid knowledge of the theories of nursing, but they are out of touch with clinical practice, and these staff members are in short supply. ${ }^{9,10}$ Studies have shown that the academic qualifications of current nurses in the clinic are generally poor, and they have limited theoretical knowledge compared to that of the specialized teachers. However, nursing is a practical and application-oriented discipline. Thereby, more clinical nurses with higher academic qualifications, better nursing skills, and better comprehensive quality should be encouraged to become nursing educators. As clinical nurses, they innately have 
some core qualities that qualify them as great teachers, such as the ability to keenly observe a situation, perform medical treatment, provide life support, ensure safety and security, conduct health education, provide human care, and perform technical operations.

There are many advantages to recruiting clinical nurses to join the nursing education faculty team. ${ }^{10}$ First, clinical nurses can introduce the latest technology and more advanced knowledge from the first line of clinical practice into the content of teaching so that the phenomenon of education lagging behind clinical practice can be changed. To a certain extent, clinical nurses can also add new elements to the connection between theoretical knowledge and clinical practice. Second, clinical nurses have long been engaged in clinical work and have a wealth of clinical experience. In teaching professional knowledge, they can use personal examples from their own work to explain the theories, thus helping the student better understand and promote more active thinking. For example, to explain the measures adopted in nursing care, by combining clinical cases, the teacher can simplify concepts that may appear boring and difficult for the students to remember; they can also assist the student in making connections between textbook knowledge and clinical practice in real life. However, clinical nurses lack the clinical experience of doctors; therefore, she or he will not be able cultivate the students' diagnostic thinking or simplify the textbook concepts for application in clinical practice. The teaching may lead to the students' systemic disconnection from the entire disease that they have learned. Third, every nursing student will be in clinical contact with patients in the future. In clinical settings, nurse-patient communication and humanistic care are essential core qualities that reflect nurses' competency. Specialized nursing teachers can only teach textbook theories to students, whereas clinical nurse faculties can use the actual case studies encountered in their work to explain the theories to the students. Consequently, the students can recognize the importance of having active communication and providing personal care to the patient and appreciate the immense value of the work performed by each nurse or nursing student. Most of the nursing educators in the United States and other countries are senior practitioners in clinical care.

\subsubsection{Teaching format}

The traditional nursing education model is a singular model. It emphasizes the transfer of knowledge and is accustomed to methods such as "injection" or "full house irrigation", which are passive methods of placing knowledge into students' brains. ${ }^{11}$ The single indoctrination mode of "teacher speaking, student listening" is commonly accepted by students. In the classroom, teachers teach textbook knowledge either in strict accordance with the textbook or in combination with some of their own cases while students merely listen. Most of the teachers complete the task mechanically and rarely ask the students any questions or initiate any interactions or discussions. The students, conversely, are often confused, and their minds shut down, not allowing them to think independently or creatively. Naturally, given our large population, adopting some teaching methods used by well-developed countries with much lower teacher-to-student ratios will be difficult. However, we can still select some educational approaches that are suitable for the situations in our country, for instance, "discovery teaching", in which the teachers guide the students to discover, explore, and solve problems to drive everyone's interest and lead the students to acquire knowledge through active learning, thinking, and participation.

\subsection{Current status of clinical practice}

\subsubsection{The disconnection between theory and practice}

Currently, the 4-year undergraduate degree in nursing science education in China is mainly composed of a 3-year theoretical study and a subsequent clinical internship for at least 8 months. In this type of curriculum arrangement, connections between the textbook knowledge and clinical practice are weak, and knowledge learned from the textbooks is not applied to clinical practice in a timely manner. ${ }^{12}$ Because of the long time interval between theoretical study and clinical practice, the textbook theories are not further reinforced through practice quickly enough. Consequently, when the clinical internship begins, the knowledge learned before has faded and fails to be adopted into clinical practice. Furthermore, during clinical practice, the nursing students only learn ward care and professional skills required by the internship department and do not take the initiative to study the theories again to further comprehend the knowledge learned in the classes. Conversely, in Australia and some other countries, nursing students complete a clinical internship after the end of each semester and also undergo a rigorous internship evaluation.

\subsubsection{Rigid training objectives in clinical internship}

Although the Chinese clinical nursing model has shifted from a functional nursing care model to a holistic nursing care model, the number of clinical nurses still only matches the functional nursing model, resulting in a 
shortage of nursing professionals. Moreover, the large number of nursing interns requires more teaching members; thus, some nurses who are not well-experienced or qualified began to conduct clinical teaching to these interns. Because of their heavy workload and lack of fundamental knowledge in theories, these nurses can only show the interns how to perform rigid procedures of care but not with critical and comprehensive thinking during the process, which causes the nursing students to repeat the daily mechanical execution of the doctor's orders. Hence, the clinical work procedures are never truly mastered during the 8 -month internship. ${ }^{13}$

\subsubsection{Lack of evidence-based clinical practice}

Evidence-based thinking and executing ability are the new objectives in the development of clinical nursing education. Presently, evidence-based nursing care is a new trend in the development of nursing science in the international nursing community. Its scientific and practical features have attracted broad attention from many domestic nursing staff members. However, evidencebased nursing is still in its infancy in China. Because clinical nurses have very limited knowledge and experience in evidence-based nursing, its teaching in clinical practice remains significantly behind that of other developed countries. Our clinical nursing care is still conducted at a simple and mechanically repeating level. Conversely, in some developed countries, evidencebased medicine is not only adopted in clinical nursing care but also applied to the investigative studies of the psychological health of nurses for nursing management and nursing research.

\subsubsection{The shortage of clinical staff and the incompletely accomplished functions of nurses}

According to the statistics of the World Health Organization and the National Health Bureau, the bed-to-nurse ratio in China still fails to meet the standard guidelines. Clinical nurses should be able to provide care services in areas, such as life care, health education, safety and security, human care, condition observation, medical treatment, and so on. However, because of the nurse shortage, each nurse is responsible for more than a reasonable number of patients. With such a heavy workload, the nursing staff only handle work that requires little time to complete, such as observing the patient's condition, and have no time to provide psychological or other forms of care. This leads to the simplification of the job function and failure to truly appreciate the value of the nursing staff and the characteristics of nursing disciplines.

\section{The developing trend of the nursing discipline}

After more than a century of development, particularly in some Western countries, nursing has become an independently developed discipline that is associated with medicine yet also distinctive from medicine. From nursing education to nursing management and from nursing research to clinical nursing care, nursing science has established a complete and independent knowledge system in its own entirety.

Studies have shown that the characteristics of a matured discipline should include a sound theoretical system, mature research methods, and specific research objects. To become an independent discipline, nursing must have developed the following: a specific philosophical foundation, subject-specific values and beliefs, conceptual systems, research methods, and a field of knowledge. $\mathrm{Li}^{14}$ has demonstrated that the key differences between the nursing discipline and other disciplines are a clear philosophical foundation, a conceptual framework that describes exactly what nursing care is, an accepted methodological development of care, and content associated with social behaviors.

Currently, people still lack a clear understanding of the relationship between nursing and medicine. The nursing model evolves with the development of the medical model, and nursing is a subdivision of medicine. The advantages of teaching nursing under the medical model, as mentioned above, also cause people to consider the nursing discipline as a part of medicine and as a field that cannot develop or think independently. However, the independent development and cogitation of a discipline must be based on the inherent relationship of the discipline with its original field. The independent development of nursing first requires reorganization, in which care and medicine are interdependent and there is an accurate understanding of the philosophical relationship between the two factors. If the independent development of the nursing discipline indicates that there is no connection between nursing and medicine, this idea is contradictory to fundamental philosophical principles. Without medicine, nursing will not exist, let alone develop independently. Some Chinese scholars believe that the formation of an independent discipline should have the following three characteristics: the discipline must have specific and other irreplaceable research objects and fields; there must be a unique theoretical system and research methods; and judging if a subject is independent or mature is dependent upon its characteristics in both theory and practice. ${ }^{14}$

Based on the above standards for the maturity of disciplines and the existing problems in nursing, we 
propose the following changes in the independent development of the nursing discipline in the future: (1) reform the nursing education curriculum, add humanities courses, and emphasize learning the diagnostics and statistics; the curriculum and teaching objectives should be coordinated; (2) improve the nursing educational system and clinical practice evaluation system; (3) follow guidelines by national policies and future social needs, increase the number of clinical nurses, and train more specialist nurses, community nurses, home nurses, and elderly nurses who will fill practical social needs; (4) integrate practice into education and provide internship opportunities soon after completing theoretical study to ensure that practice and theory are closely connected; (5) enhance the team of nursing faculties and improve the academic level of nursing disciplines; and (6) innovate consistently to build a nursing theory system based on philosophical thinking.

\section{References}

1. Engel GL. The need for a new medical model: a challenge for biomedicine. Science. 1977;196:129-136.

2. Ying $Y$. Discussion on how to distinguish health assessments from diagnostics in teaching practice. J Shanxi Coll Continuing Educ. 2013;23:64-66 (in Chinese).

3. Sha YH, Chen ZY. A discussion on the correlation between Health Assessment, Diagnostics and Clinical Nursing Courses. Health Vocational Educ. 2009;27:126-127 (in Chinese).

4. Ren J, Jiang YG. Problems and countermeasures in humanity education in nursing education. China Med Pharm. 2012;2:142-144 (in Chinese).

5. Guo HX, Chen H. Analysis of the Danish nursing undergraduate curriculum setting and enlightenment. West China Med J. 2016;31:1926-1929 (in Chinese).

6. Ouyang L, Liu J. Highlights from Australian nursing education development and enlightenment on nursing education in China. China Health Ind. 2017;23:104-105 (in Chinese).

7. Li LX, Jiang ZX, Zheng XL. Training goal for nursing undergraduates: a qualitative study. J Nurs Sci. 2012;27:80-82 (in Chinese).

\section{Conclusions}

In summary, nursing is a practical science, and its research objects are all humans in society. We should use humans as study objects to identify suitable research methods for the nursing discipline and build a unique nursing theoretical system that fits our county's situation based on certain philosophies. This approach will truly allow the discipline to develop independently. In an age when health is the core focus of health care, modern nursing care must focus on the entire health chain of prevention, treatment, and rehabilitation. Establishing multidisciplinary cooperation will become the future direction of the nursing discipline.

\section{Conflicts of interest}

All contributing authors declare no conflicts of interest.

8. Jiang AL. Problems in the education plan for undergraduate professional certification in nursing and suggestions. Chin J Nurs Educ. 2016;13:491-495 (in Chinese).

9. Li C, Tu B, Ren HY. Analysis on the quality of faculty of nursing professionals in colleges and universities. Educ Teach Forum. 2013;46:33-34 (in Chinese).

10. Hou R, Zhang ZX. The composition and selection of clinical nursing mentors in China. $J$ Nurs Sci. 2013;17:88-90 (in Chinese).

11. Wang MY. Analysis of the current status and problems in the teaching methods in nursing. Nurs Pract Res. 2011;8:100-101 (in Chinese).

12. Zhao $\mathrm{XL}$. Connecting factors in nursing and clinical practice. Word Latest Med Info (Electronic version). 2016;34:288-289 (in Chinese).

13. Wang YY, Huo HM, Zhang Y. Problems with "teaching" in clinical nursing teaching practice and countermeasures. China J Pharm Econ. 2013;5:187-188 (in Chinese).

14. Li J. Exploratory Research on the Construction of the Nursing Discipline System. Shanghai: School of Nursing, Second Military Medical University. 2010:1-83 (in Chinese). 